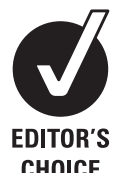

CHOICE

\section{See Editorial, p 649}

- An additional table and appendix are published online only. To view these files please visit the journal online (http:// thorax.bmj.com).

${ }^{1}$ Department of Preventive Medicine, Vanderbilt University School of Medicine, Nashville Tennessee, USA

${ }^{2}$ Department of Biostatistics, Vanderbilt University School of Medicine, Nashville, Tennessee, USA

${ }^{3}$ The National Center for Immunization and Respiratory Diseases, Centers for Disease Control and Prevention, Atlanta, Georgia, USA

${ }^{4}$ Department of Medicine Vanderbilt University School of Medicine, Nashville, Tennessee, USA

\section{Correspondence to}

Carlos G Grijalva, Department of Preventive Medicine, Vanderbilt University School of Medicine 1500 21st Avenue Suite 2600 The Village at Vanderbilt, Nashville, TN 37232-2637, USA carlos.grijalva@vanderbilt.edu

Received 22 November 2010 Accepted 28 April 2011 Published Online First 26 May 2011

\title{
Emergence of parapneumonic empyema in the USA
}

\author{
Carlos G Grijalva, ${ }^{1}$ Yuwei Zhu, ${ }^{2}$ J Pekka Nuorti, ${ }^{3}$ Marie R Griffin ${ }^{1,4}$
}

ABSTRACT
Background Although recent reports suggest that the incidence of parapneumonic empyema has increased in several regions of the USA, national trends in disease burden are unknown. National trends in the incidence of parapneumonic empyema hospitalisations and changes in empyema by associated pathogens were examined. Methods National hospitalisation data (1996-2008) were analysed and rates estimated using census estimates as denominators. Incidence rate ratios (IRR) compared 2008 with 1996 rates. Discharge diagnosis codes were used to characterise pathogens associated with empyema hospitalisations.

Results Overall, national parapneumonic empyemarelated hospitalisation rates increased from 3.04 per 100000 in 1996 to 5.98 per 100000 in 2008, a 2.0-fold increase $(95 \% \mathrm{Cl} 1.8$ to 2.1$)$. The increases were observed among children (IRR 1.9 (95\% Cl 1.4 to 2.7)) and adults aged 18-39, 40-64 and $\geq 65$ years (IRR 1.8 (95\% Cl 1.5 to 2.1), 2.0 (95\% Cl 1.6 to 3.1$)$ and $1.7(95 \%$ Cl 1.5 to 2.0), respectively). Overall, pneumococcal empyema rates remained relatively stable in all age groups whereas streptococcal- (non-pneumococcal) and staphylococcal-related empyema rates increased 1.9-fold and 3.3-fold, respectively, with consistent increases across age groups. The overall in-hospital case fatality ratio for parapneumonic empyema-related hospitalisations was $8.0 \%$ (95\% $\mathrm{Cl} 6.4 \%$ to $9.5 \%$ ) in 1996 and $7.2 \%(95 \% \mathrm{Cl} 6.3 \%$ to $8.1 \%)$ in 2008 $(p=0.395)$. Of the empyemas where study pathogens were listed (37.6\%), staphylococcal-related empyema had the largest absolute increases across age groups and was associated with longer hospital stay and higher in-hospital mortality than other empyemas.

Conclusions Although parapneumonic empyemarelated hospitalisations remained relatively rare, they increased substantially during the study period. A number of pathogens, especially staphylococcus, contributed to this increase.

\section{INTRODUCTION}

Empyema is a relatively rare but serious infection of the pleural space. Despite the wide availability of novel antibiotics and improved diagnostic tools, empyema remains an important cause of morbidity and mortality. ${ }^{1}$ Delays in diagnosis, failure to institute appropriate antimicrobial therapy and inadequate drainage contribute to increased morbidity and mortality. ${ }^{1-4}$ In addition, the inhospital management of patients with empyema is associated with substantial costs. ${ }^{5}$

The pathogens traditionally associated with empyema are Streptococcus pneumoniae, Streptococcus pyogenes and Staphylococcus aureus. ${ }^{1}$ However, anaerobes have also been identified in $25-76 \%$ of cases as sole organisms or mixed with other

\section{Key messages}

What is the key question?

This study examined changes in the incidence of parapneumonic empyema hospitalisations and associated pathogens in the USA.

What is the bottom line?

Parapneumonic empyema-related hospitalisations have increased substantially during the last years. A number of pathogens, especially staphylococcus, contributed to this increase.

\section{Why read on?}

Empyema is a relatively rare yet very serious disease. This article describes recent increases in its incidence and highlights the need for preventive strategies.

bacteria. ${ }^{1}{ }^{6}$ Recently, the Streptococcus anginosus (formerly termed Streptococcus milleri) ${ }^{7}$ group has been reported as a leading bacterial cause of empyema. ${ }^{13} 8$ Approximately $40-60 \%$ of empyemas are considered to be pneumonia-related (ie, parapneumonic), whereas thoracic procedures and trauma account for most of the remainder. ${ }^{1}$ Most published descriptions of empyema come from reports from large healthcare referral centres.

Previous reports suggested that the incidence of parapneumonic empyema was increasing in several regions of the USA ${ }^{6-11}$ and in other countries. $^{4}{ }^{12}{ }^{13}$ More recently, studies have shown national increases in childhood empyema and parapneumonic empyema in young children, with various pathogens contributing to these increases. ${ }^{14-16}$ However, there have been no comprehensive studies of US trends in parapneumonic empyemas including both children and adults. We used national hospitalisation data to examine the patient characteristics and trends of parapneumonic empyema-related hospitalisations in the USA.

\section{METHODS}

\section{Source of data}

National hospitalisation data were obtained from the Nationwide Inpatient Sample (NIS), which is the largest source of inpatient data publicly available in the USA. These databases contain information on inpatient stays from states that participate in the Healthcare Cost and Utilisation Project (HCUP), sponsored by the Agency for Healthcare Research and Quality. Information recorded includes clinical and resource utilisation data for 5-8 million hospitalisations per year from a sample of approximately 1000 hospitals. These hospitals constitute an estimated 20\% sample 
of community hospitals, including non-federal short-term, general and specialty hospitals. Participating hospitals are sampled by stratified probability sampling in five strata (ownership/control, bed size, teaching status, urban/rural and region), with sampling probabilities proportional to the number of community hospitals in each stratum. ${ }^{17}$

NIS collects data on all hospitalisations regardless of payment source, and weighting and sampling variables are provided to calculate national estimates. Up to 15 discharge diagnoses and procedures are recorded using ICD9-CM codes with first-listed diagnoses (principal) regarded as the primary reason for hospitalisation. ${ }^{18}$ NIS data are publicly available and have no personal identifiers. This study was considered exempt from review by the institutional review boards of Vanderbilt University and the Centers for Disease Control.

\section{Empyema identification}

We identified parapneumonic empyema hospitalisations using ICD9-CM coded discharge diagnoses. ${ }^{15}$ We first identified allcause pneumonia hospitalisations by a principal diagnosis of pneumonia, or a principal diagnosis of septicaemia, meningitis or empyema and a pneumonia code in another diagnosis field. ${ }^{15} 19$ Discharge diagnosis and procedure codes, including thoracentesis and related procedures (including video-assisted thoracoscopic surgery, VATS), ${ }^{5}$ were then used to characterise parapneumonic empyema hospitalisations (online appendix). About $9 \%$ of parapneumonic empyema hospitalisations had a primary diagnosis of sepsis/septicaemia or meningitis.

\section{Statistical analyses}

Annual incidence rates of parapneumonic empyema hospitalisations were calculated by dividing the weighted number of empyemas by annual US census population estimates. Diagnosis codes indicating specific aetiologies for empyema-related pathogens (ie, specified pneumonia, septicaemia/bacteraemia or specific infections) were used to classify empyemas into mutually exclusive groups based on the following hierarchy: (1) pneumococcal; (2) streptococcal (non-pneumococcal); (3) staphylococcal; and (4) other/unknown pathogens.

Poisson regression models with terms for age group, year and one age-time term interaction were fit for each empyema group to obtain annual incidence rates, incidence rate ratios (IRRs) and variance estimates while accounting for the NIS sampling design. The unit of analysis was the empyema hospitalisation and the offset term for these count-events models were the census population estimates for specific years and age groups. Thus, for the calculation of incidence rates, the aggregated NIS weighted estimates were divided by their respective population estimates. All analyses accounted for the NIS weight (DISCWT), stratum (NIS_STRATUM and STRATUM) and cluster (HOSPID) design variables. Changes in annual empyema rates were examined and summarised by estimating the IRR of the last (2008) to the first (1996) annual rates available. Rates, IRRs and their respective 95\% CIs were calculated using linear combination of coefficients from the fitted Poisson models. In addition, rate differences were calculated and tests for linear trends were performed evaluating the effect of calendar year on the estimated rates. Mean length of stay, number of discharge diagnoses and number of procedures were compared using t tests that accounted for the NIS sampling design. In-hospital case fatality ratios were estimated by dividing the number of inhospital fatalities by the number of empyema-related hospitalisations. Since previous empyema studies have focused on young children aged $<5$ years, ${ }^{14}{ }^{15}$ the analyses for the present study were age stratified as $0-17,18-39,40-64$ and $\geq 65$ years.

To assess the potential effect of changing coding practices on the observed trends, we assessed changes in the mean number of discharge diagnoses, procedures and length of stay (in days) for allcause hospitalisations and for parapneumonic empyema-related hospitalisations.

Secondary analyses aimed to exclude hospital-acquired empyema and were restricted to those patients with a primary discharge diagnosis of empyema. Statistical analyses used the survey packages SAS 9.2 and Stata 11.0.

\section{RESULTS \\ Characteristics of parapneumonic empyema-related hospitalisations}

From 1996 through 2008 we estimated 157094 parapneumonic empyema-related hospitalisations in the USA (18200 in 2008). The mean patient age was 48.4 years (95\% CI 47.5 to 49.4 ) and $35.8 \%$ were female. Race/ethnicity was not reported in $23.7 \%$ and was reported as white, African-American, Hispanic and other in $56.1 \%, 8.8 \%, 6.9 \%$ and $4.7 \%$, respectively (table 1 ).

Thoracentesis and related procedures were recorded in $86.2 \%$ of all parapneumonic empyema hospitalisations, and the proportion with any of these procedures was higher among children $(84.6 \%)$ than among adults aged $\geq 65$ years $(81.6 \%$ ). The mean length of stay was 15.2 days and was shorter for children than for adults. The overall in-hospital case fatality

Table 1 Characteristics of parapneumonic empyema-related hospitalisations in the USA, 1996-2008*

\begin{tabular}{|c|c|c|c|c|c|}
\hline & $<18$ years $(n=26125)$ & $18-39$ years $(n=22185)$ & $40-64$ years $(n=62358)$ & $\geq 65$ years $(n=46375)$ & All $(n=157094)$ \\
\hline Age, years & 5.1 (5 to 5.3$)$ & 30.7 (30.6 to 30.9$)$ & 51.9 (51.8 to 52$)$ & 76.7 (76.5 to 76.8 ) & 48.4 (47.5 to 49.4 ) \\
\hline Female, \% & 44.7 (43.4 to 46$)$ & 30.2 (28.8 to 31.6$)$ & 30.8 (30 to 31.7$)$ & 40.1 (39.1 to 41.2 ) & $35.8(35.2$ to 36.4$)$ \\
\hline \multicolumn{6}{|l|}{ Race, \% } \\
\hline White & 42 (38.7 to 45.3$)$ & 52 (49.8 to 54.3 ) & 56.7 (54.8 to 58.6$)$ & 65.3 (63.3 to 67.2 ) & 56.1 (54.4 to 57.8 ) \\
\hline African-American & $11.4(9.3$ to 13.4$)$ & $10.9(9.7$ to 12.1$)$ & 9.7 (8.8 to 10.6$)$ & $5.1(4.5$ to 5.7$)$ & $8.8(8.0$ to 9.5$)$ \\
\hline Hispanic & $13.1(10.8$ to 15.4$)$ & $9.2(8$ to 10.4$)$ & $5.9(5.2$ to 6.5$)$ & $3.6(3.1$ to 4.1$)$ & $6.9(6.2$ to 7.6$)$ \\
\hline Asian or Pacific Islander & $3.7(2.8$ to 4.5$)$ & 1.6 (1.2 to 2$)$ & $1.4(1.1$ to 1.7$)$ & 1.8 (1.4 to 2.2$)$ & $1.9(1.7$ to 2.2$)$ \\
\hline Native American & $0.8(0.4$ to 1.2$)$ & $0.7(0.3$ to 1$)$ & $0.4(0.3$ to 0.6$)$ & $0.3(0.1$ to 0.4$)$ & $0.5(0.3$ to 0.6$)$ \\
\hline Other & 4.5 (3.6 to 5.4$)$ & $2(1.6$ to 2.5$)$ & 1.8 (1.5 to 2.1$)$ & 1.5 (1.2 to 1.8$)$ & $2.2(1.9$ to 2.5$)$ \\
\hline Missing & 23.6 (18.5 to 28.6$)$ & $24.1(21.3$ to 27.0$)$ & $24.5(22.2$ to 26.9$)$ & $23.0(20.7$ to 25.3$)$ & 23.7 (21.7 to 25.7$)$ \\
\hline Length of stay, days & $11.8(11.4$ to 12.2$)$ & $14.4(14$ to 14.8$)$ & $15.6(15.4$ to 15.9$)$ & $16.8(16.4$ to 17.1$)$ & $15.2(15.0$ to 15.4$)$ \\
\hline Thoracentesis†, \% & $84.6(82.7$ to 86.5$)$ & $91.3(90.4$ to 92.2 ) & 88.4 (87.7 to 89$)$ & 81.6 (80.8 to 82.5 ) & 86.2 (85.6 to 86.7 ) \\
\hline In-hospital case fatality, \% & $0.4(0.3$ to 0.6$)$ & $2.2(1.8$ to 2.7$)$ & $5.2(4.8$ to 5.6$)$ & $16.1(15.3$ to 16.9$)$ & 7.2 (6.8 to 7.5$)$ \\
\hline
\end{tabular}

*Numbers indicate percentages and $95 \%$ Cls unless otherwise specified. Numbers may not add to totals or $100 \%$ due to rounding/missing values.

†Includes thoracentesis-related procedures (ICD9-CM: 34.04, 34.06, 34.09, 34.21, 34.51, 34.52, 34.91). 
ratio was $7.2 \%$, ranging from $0.4 \%$ in children to $16.1 \%$ in adults aged $\geq 65$ years (table 1 ).

\section{Assessment of coding practices}

During the study years the mean length of all-cause hospital stay decreased from 5.0 days in 1996 to 4.6 days in 2008 $(p<0.001)$. Similarly, the mean length of hospital stay for parapneumonic empyema-related hospitalisations declined from 16.5 days (95\% CI 15.7 to 17.2 ) in 1996 to 14.9 days (95\% CI 14.4 to 15.4$)$ in $2008(\mathrm{p}<0.001)$.

For all-cause hospitalisations there was an increase in the overall mean number of discharge diagnoses recorded from 4.5 in 1996 to 7.3 in $2008(p<0.001)$. The mean number of discharge diagnoses recorded during parapneumonic empyema-related hospitalisations also increased from $7.2(95 \%$ CI 7.0 to 7.4$)$ in 1996 to 11.8 (95\% CI 11.4 to 12.2$)$ in 2008 ( $p<0.001)$.

The mean number of procedures recorded during all-cause hospitalisations increased from 1.4 to $1.6(p<0.001)$ during the study years. Similarly, the mean number of procedures performed during parapneumonic empyema hospitalisations increased from 4.0 (95\% CI 3.8 to 4.3 ) in 1996 to 4.5 (95\% CI 4.3 to 4.7$)$ in $2008(p=0.002)$. The five most frequent procedures listed as first procedures among parapneumonic empyema hospitalisations during 2008 included: insertion of intercostal cathether, decortication of the lung, thoracoscopic decortication of the lung, thoracentesis and mechanical ventilation.

\section{Trends in the incidence of parapneumonic empyema hospitalisations}

The mean annual rate of parapneumonic empyema hospitalisations varied with age, with the largest burden concentrated in the elderly. Mean rates for children were 2.76 per 100000 (95\% CI 2.49 to 3.07), whereas mean annual rates for adults aged $18-39,40-64$ and $\geq 65$ years were 1.89 (95\% CI 1.82 to 1.97$)$, 5.41 (95\% CI 5.28 to 5.56 ) and 9.94 (95\% CI 9.68 to 10.21), respectively. Overall, national rates of parapneumonic empyema hospitalisations increased from 3.04 per 100000 in 1996 to 5.98 per 100000 in 2008, a 2.0-fold increase (95\% CI 1.8 to 2.1, test for trend $\mathrm{p}<0.001)$. In the age-stratified analysis the largest relative increase was observed among adults aged 40-64 years, with rates increasing from 3.96 per 100000 in 1996 to 8.10 per 100000 in 2008, representing a 2.0-fold increase (95\% CI 1.6 to 3.1 , test for trend $\mathrm{p}<0.001$ ) (figure 1 ).

Parapneumonic empyema hospitalisations also increased among other age groups. In children the rates increased 1.9-fold

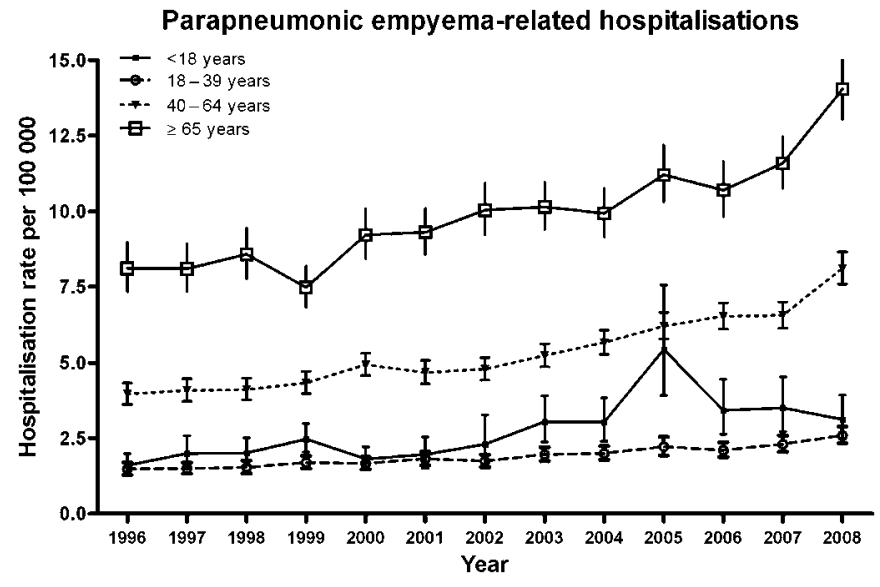

Figure 1 Trends in parapneumonic empyema-related hospitalisations in the USA, 1996-2008. Data from the Nationwide Inpatient Sample.
(95\% CI 1.4 to 2.7 , test for trend $\mathrm{p}<0.001)$. Similarly, the rates increased 1.8-fold (95\% CI 1.5 to 2.1 , test for trend $p<0.001$ ) and 1.7 -fold (95\% CI 1.5 to 2.0 , test for trend $\mathrm{p}<0.001$ ) among adults aged $18-39$ years and $\geq 65$ years, respectively (figure 1 ).

The overall in-hospital case fatality ratio for parapneumonic empyema hospitalisations was $8.0 \%$ (95\% CI $6.4 \%$ to $9.5 \%$ ) in 1996 and $7.2 \%$ (95\% CI $6.3 \%$ to $8.1 \%)$ in $2008(\mathrm{p}=0.395)$. The rate of fatal parapneumonic empyema hospitalisations increased from 0.24 (95\% CI 0.20 to 0.30 ) per 100000 in 1996 to 0.43 (95\% CI 0.38 to 0.49$)$ per 100000 in 2008, a 1.8-fold increase (95\% CI 1.4 to 2.3 , test for trend $\mathrm{p}<0.001$ ).

\section{Empyema related to specific pathogens}

Overall, the rates of pneumococcal empyema-related hospitalisations were 0.68 and 0.74 per 100000 in 1996 and 2008, respectively (rate ratio 1.1 (95\% CI 0.9 to 1.3 , test for trend $\mathrm{p}=0.433$ ). Pneumococcal empyema rates remained stable among children (rate ratio 1.1, 95\% CI 0.7 to $1.7, \mathrm{p}$ value for test for trend $\mathrm{p}=0.093$ ) and adults (rate ratios 1.3, 1.2 and 0.8 for adults aged $18-39,40-64$ and $\geq 65$ years, respectively).

Rates of streptococcal (non-pneumococcal) empyema increased 1.9-fold (95\% CI 1.6 to 2.4 , test for trend $p<0.001$ ) during the study period, whereas rates of staphylococcal empyema increased by 3.3 -fold $(95 \%$ CI 2.7 to 4.0 , test for trend $p<0.001$ ) (figure 2). The mean length of hospitalisations was also consistently longer for staphylococcal empyema than for empyema associated with other pathogens and across age groups. Although the frequency of thoracentesis and related procedures was similar among all aetiological groups of empyema, the overall in-hospital case fatality ratio was higher among those with staphylococcal empyema compared with other groups (table 2).

Of the parapneumonic empyema hospitalisations, $62.4 \%$ were listed as having other/unknown aetiology. The proportion of other/unknown aetiology empyema increased with age and the rates of these empyemas increased 2.1-fold (95\% CI 1.9 to 2.3, test for trend $\mathrm{p}<0.001$ ) (figure 2 ). (Additional information available in online table).

In the secondary analysis restricted to patients with empyema listed as the primary discharge diagnosis (45\% of all parapneumonic empyema), the mean length of stay was 14.0 days (95\% CI 13.7 to 14.2$)$ and the case fatality ratio was 5.8\% (95\% CI $5.4 \%$ to $6.2 \%$ ), both lower than those observed for all parapneumonic empyemas. In this subgroup the overall rates of empyema hospitalisations increased 1.9-fold (95\% CI 1.6 to 2.1). The increase in children was 1.9-fold, and 1.7, 2.0 and 1.5 among adults aged $18-39,40-64$ and $\geq 65$ years, respectively. Consistent with the results from the main analyses, overall rates of pneumococcal empyema remained stable (IRR 0.9 (95\% CI 0.6 to 1.2)) whereas rates of streptococcal (non-pneumococcal), staphylococcal and other empyema increased 1.7-, 2.9- and 2.0-fold, respectively.

\section{DISCUSSION}

National hospitalisation data collected over a 13-year period were used to assess changes in the epidemiology of parapneumonic empyema. Our study revealed a substantial increase in the incidence of parapneumonic empyema hospitalisations in the USA. Parapneumonic empyema hospitalisations were commonly associated with in-hospital mortality and required long hospital stays. The largest relative increases were observed among young adults; however, the largest absolute increases occurred in older populations. Our data suggest that the most serious diseases were related to staphylococcal infections. 


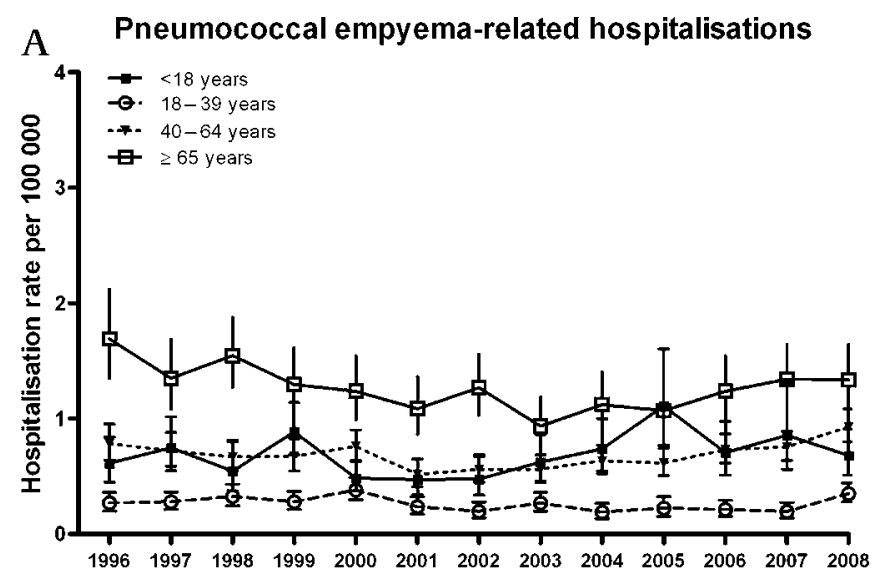

B Streptococcal (non pneumococcal) empyema-related hospitalisations
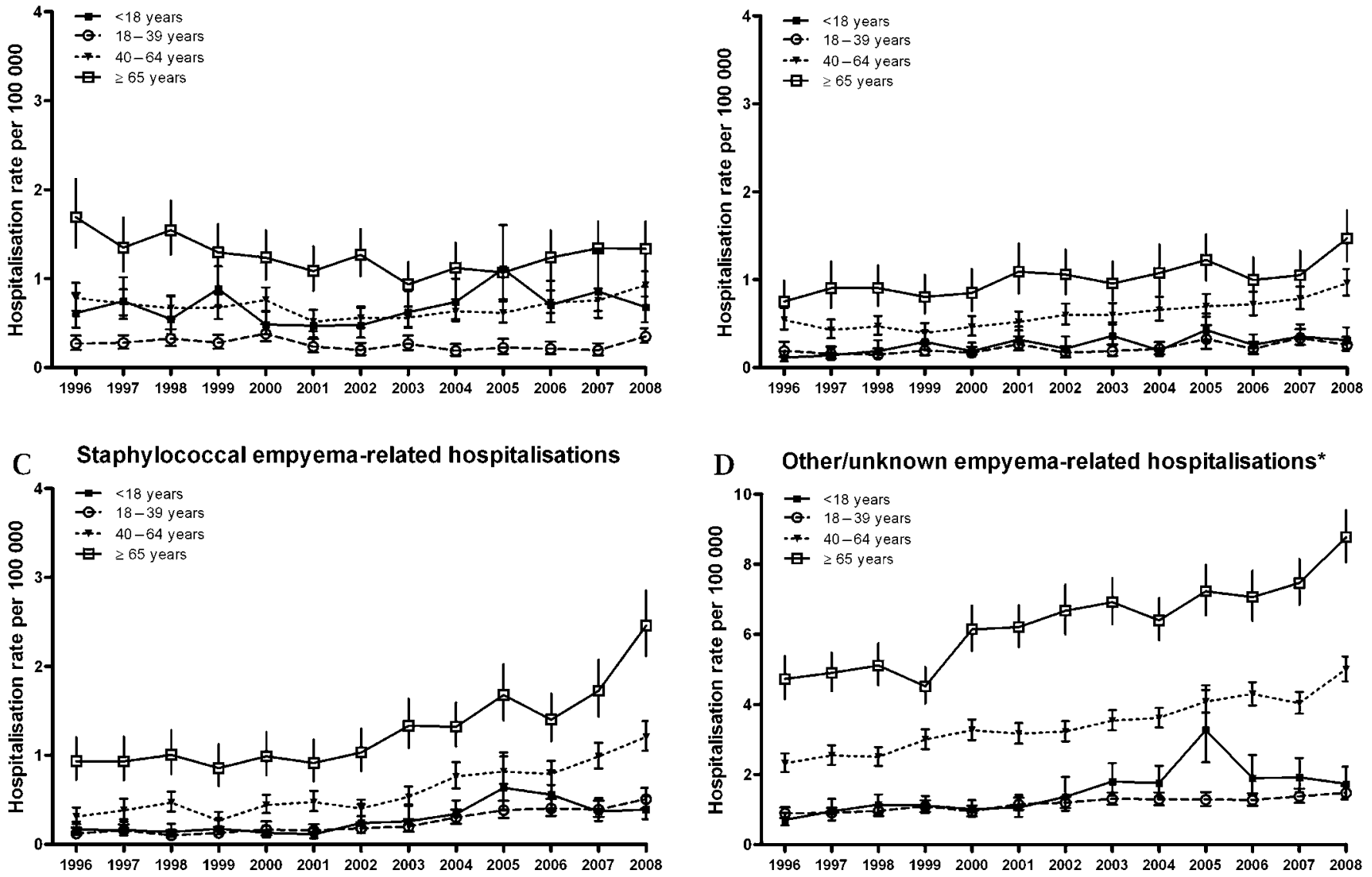

Figure 2 (A-D) Trends in parapneumonic empyema-related hospitalisations by different pathogens in the USA, 1996-2008. Data from the Nationwide Inpatient Sample. Note the scale change for estimates of other/unknown empyema.

Although parapneumonic empyema remains a relatively rare occurrence among children and young adults, empyema was more common and associated with a high in-hospital case fatality ratio among elderly people. The observed increasing trend is worrisome and consistent with previous reports from large healthcare centres or regions within the USA and abroad. ${ }^{4} 6$ 9-13

Although most empyemas had other/unknown aetiology, many pathogens appear to contribute to the observed increase.

Table 2 Selected characteristics of empyema-related hospitalisations by age group and associated pathogens in the USA, $1996-2008$

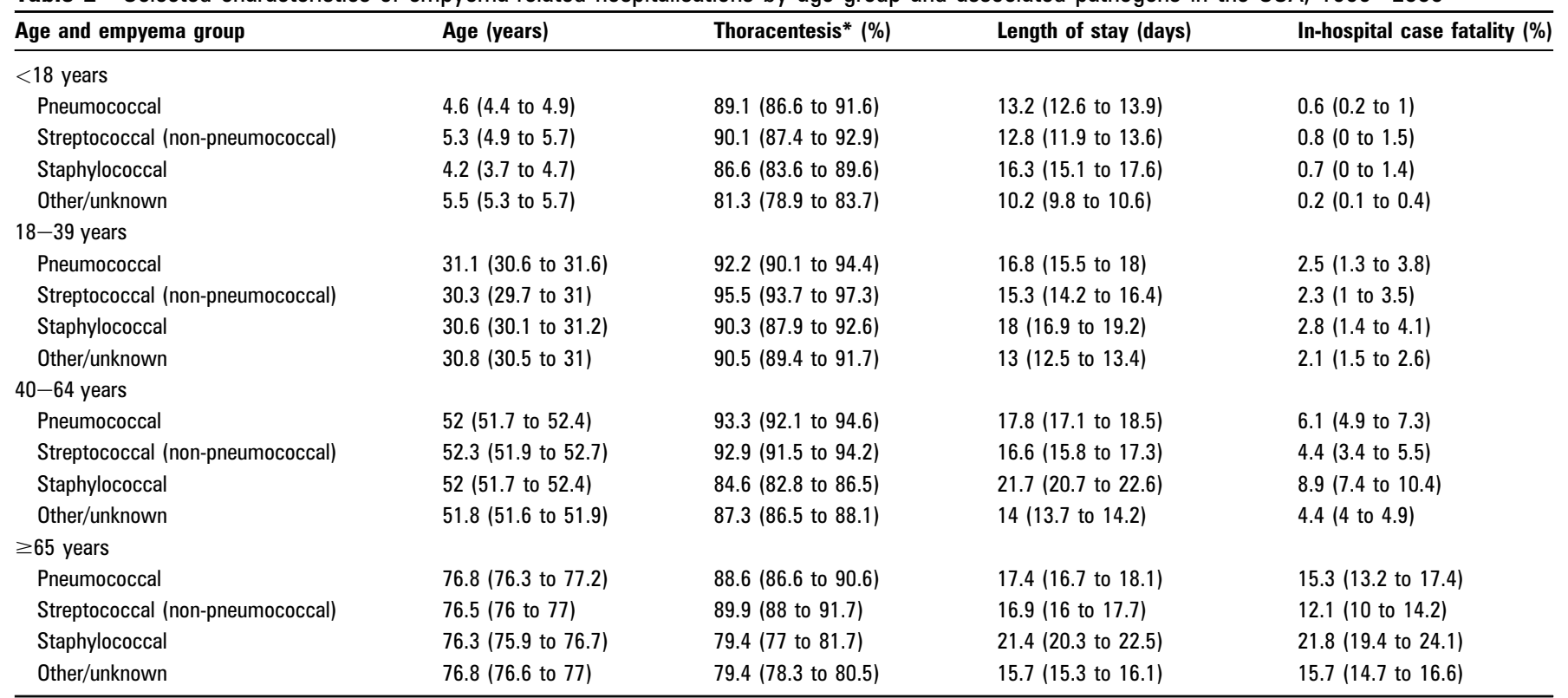

Values shown are mean $(95 \% \mathrm{Cl})$, unless otherwise specified.

*Includes thoracentesis-related procedures (ICD9-CM: 34.04, 34.06, 34.09, 34.21, 34.51, 34.52, 34.91). 
Among cases classified in our study as pathogen-specific groups, staphylococcal related-empyema accounted for most of the increase observed during the study years and these patients had the longest lengths of stay and the highest in-hospital case fatalities, suggesting more severe disease than other empyemas. The observed national increases in empyema associated with staphylococcal infections are also consistent with previous studies performed in large referral healthcare centres. ${ }^{6} 20$

In contrast with the increases observed in other empyema groups, the incidence of pneumococcal empyema remained stable during the study period in both children and adults. It is possible that stable rates of pneumococcal empyema hospitalisations were related to direct and indirect protection derived from infant vaccination with a seven-valent pneumococcal conjugate vaccine (PCV7) that started in 2000. ${ }^{21-24}$ Previous studies have documented significant reductions in the incidence of pneumonia hospitalisations after the introduction of PCV7. ${ }^{15}{ }^{19}$ Some studies reported local and regional increases in the frequency of pneumococcal empyemas in children which started in the $1990 \mathrm{~s}^{9}{ }^{25}$ and continued after 2000. ${ }^{10}{ }^{11}$ Most of these increases were related to serotypes not covered in the PCV7 formulation, most notably serotype $1 .{ }^{9} 25$ A recent study assessed national rates of pneumonia-related empyema in children aged $<5$ years and observed a significant increase in children aged 2-4 years but not in younger children. ${ }^{15}$ Another study that explored national changes in childhood pneumococcal empyema reported stable rates from 1997 through $2006 .{ }^{14}$ The present study, which was not restricted to young children, indicates that overall national rates of childhood pneumococcal empyema remained relatively stable during the study period. The recent introduction of a 13 -valent pneumococcal conjugate vaccine in 2010 in the USA could provide protection against pneumococcal serotypes $1,3,7 \mathrm{~F}$ and 19A commonly associated with empyema, ${ }^{26} 27$ but the assessment of these vaccination effects will require additional studies.

A large number of parapneumonic empyemas were classified as being caused by other or unknown pathogens and large increases were observed in this group. Defining the aetiology of empyema is difficult, and it is unclear whether these increases in empyema caused by other or unknown pathogens also indicates true emerging trends, reduced or delayed laboratory testing or increased use of antibiotics prior to hospitalisation or sample collection. Furthermore, mixed bacterial infections are found in some parapneumonic empyemas ${ }^{28}$ but detailed microbiological information was not available for the present study.

The actual cause of the observed increase in empyema is unclear. The spread of antibiotic-resistant bacteria, some of which are major empyema pathogens, may be a driver of the observed increases. It was previously suggested that the failure to initiate appropriate antibiotic treatment could result in an increased incidence of this condition. In particular we observed increases in empyema related to staphylococcal infections, which have shown an increasing prevalence of antibiotic resistance during recent years. ${ }^{4}$ The increasing incidence of infections due to antibiotic-resistant pathogens in the USA is well documented, ${ }^{29}$ with $S$ pneumoniae being the major exception as the incidence of invasive pneumococcal disease caused by resistant strains has decreased markedly following the introduction of PCV7. ${ }^{30}$

It is also possible that the increasing wide availability of new diagnostic techniques such as CT scans could have contributed to an increased recognition of empyemas and to the observed increases. ${ }^{31}$ Moreover, other changes in the detection and management of empyema developed during this period. For instance, VATS became a common procedure and studies suggest that it can reduce the length of hospital stay and improve clinical outcomes. ${ }^{5} 32$ The precise contribution of these changes in the early detection and treatment of empyemas to the observed trends is unclear. In our study the mean number of thoracentesis-related procedures performed during empyema hospitalisations increased throughout the study years. Notably, in spite of the increasing incidence of empyema observed, the mean length of hospital stay declined and the case fatality ratio associated with this condition remained stable. These findings could also be related to increased detection of less severe episodes of empyema or more complete coding of these episodes. It is also possible that there were more readmissions for empyema in recent years. Since NIS data lack personal identifiers, we did not assess the contribution of multiple admissions for the same person to the incidence rates.

Our result must be interpreted in the light of several limitations. First, we used coded discharge diagnoses to identify parapneumonic empyema and, as a systematic review of clinical cases could not be performed, some misclassification of events cannot be ruled out. Our data used de-identified information, and chart reviews or validation of the empyema discharge diagnosis was not feasible. Nevertheless, the vast majority of empyema hospitalisations had related procedures listed suggesting high specificity of the discharge diagnoses. Second, although our data provide an overview of national changes in the incidence of parapneumonic empyema hospitalisations, a detailed assessment of the clinical characteristics of the patients and their microbiological analyses was not possible. Third, our study probably included some empyemas that developed during the course of a hospitalisation (hospitalacquired), which appear to have different outcomes and aetiologies than community-acquired empyemas. ${ }^{28}$ Although our study could not specifically distinguish hospital-acquired empyemas, a secondary analysis that focused on a principal diagnosis of empyema (used as a surrogate for the main reason for hospitalisation and thus community-acquired empyema) showed results consistent with our main findings. Fourth, although NIS provides a valuable resource for the study of hospitalisations in the USA, this resource does not collect data from patients who receive their care exclusively from federal institutions such as the Veterans Affairs healthcare institutions and therefore modestly underestimates the real disease burden in adults. Finally, our data sources lacked information for the period preceding hospital admission and it is possible that some patients with empyema were admitted to hospital after a previous hospitalisation for pneumonia or pneumonia managed in the ambulatory setting. In this scenario, some empyema hospitalisations could appear unrelated to pneumonia and would be excluded, leading to an underestimation of the burden of parapneumonic empyema.

The aetiology of empyema is difficult to establish and we used available information to assess aetiologies. Some studies used molecular techniques and suggested that a sizeable proportion of culture-negative empyema was caused by pneumococci, mainly serotype $1^{12}$ 33-36 Moreover, although recent studies described the Streptococcus anginosus group as a leading bacterial cause of empyema, ${ }^{3} 828$ our ability to discriminate between streptococcal species was limited and we evaluated streptococcal (non-pneumococcal) empyema as a single group. Moreover, for a large proportion of patients (particularly older adults), empyema was recorded as of other/unknown aetiology.

In conclusion, our study showed a substantial increase in the incidence of parapneumonic empyema hospitalisations in the 
USA. Compared with other types of empyema, staphylococcalrelated empyema showed the overall largest relative increase during the study period and was associated with relatively longer hospital stays and higher mortality.

Funding This study was funded by the Centers for Disease Control and Prevention through a Cooperative Agreement with the Association for Prevention Teaching and Research (TS-1454). CGG is supported by a CDC career development award (K01 Cl000163).

Competing interests CGG received lecture fees from Wyeth (now Pfizer) and has been a consultant for GlaxoSmithKline. MRG received grant support from Med Immune. CGG and MRG received research support for an investigator-originated project from Wyeth (now Pfizer).

Ethics approval This study was considered exempt from review by the institutional review boards of Vanderbilt University and the Centers for Disease Control.

Contributors Study concept and design: CGG, MRG; acquisition of data: CGG; analysis and interpretation of data: CGG, YZ, JPN, MRG; drafting of the manuscript: CGG, MRG; critical revision of the manuscript for important intellectual content: CGG, YZ, JPN, MRG; statistical analysis: CGG, YZ; obtained funding: CGG, MRG; study supervision: CGG, JPN, MRG.

Provenance and peer review Not commissioned; externally peer reviewed.

\section{REFERENCES}

1. Septimus E. Pleural effusion and empyema. In: Mandell GL, Bennett JE RD, eds. Mandell, Douglas, and Bennett's Principles and Practice of Infectious Diseases. Edinburgh: Churchill Livingstone, 2009:917-24.

2. Ashbaugh DG. Empyema thoracis. Factors influencing morbidity and mortality. Chest 1991:99:1162-5.

3. Ahmed RA, Marrie TJ, Huang J0. Thoracic empyema in patients with communityacquired pneumonia. Am J Med 2006;119:877-83.

4. Finley C, Clifton J, Fitzgerald JM, et al. Empyema: an increasing concern in Canada. Can Respir J 2008;15:85-9.

5. Li ST, Gates RL. Primary operative management for pediatric empyema: decreases in hospital length of stay and charges in a national sample. Arch Pediatr Adolesc Med 2008;162:44-8.

6. Schultz KD, Fan LL, Pinsky J, et al. The changing face of pleural empyemas in children: epidemiology and management. Pediatrics 2004;113:1735-40.

7. Ruoff KL. Streptococcus anginosus ("Streptococcus milleri"): the unrecognized pathogen. Clin Microbiol Rev 1988:1:102-8.

8. Maskell NA, Davies CW, Nunn AJ, et al. UK controlled trial of intrapleural streptokinase for pleural infection. N Engl J Med 2005;352:865-74.

9. Byington CL, Spencer LY, Johnson TA, et al. An epidemiological investigation of a sustained high rate of pediatric parapneumonic empyema: risk factors and microbiological associations. Clin Infect Dis 2002;34:434-40.

10. Byington CL, Samore MH, Stoddard GJ, et al. Temporal trends of invasive disease due to Streptococcus pneumoniae among children in the Intermountain West: emergence of nonvaccine serogroups. Clin Infect Dis 2005:41:21-9.

11. Byington $\mathbf{C L}$, Korgenski $\mathrm{K}$, Daly J, et al. Impact of the pneumococcal conjugate vaccine on pneumococcal parapneumonic empyema. Pediatr Infect Dis $J$ 2006:25:250-4.

12. Obando I, Munoz-Almagro C, Arroyo LA, et al. Pediatric parapneumonic empyema, Spain. Emerg Infect Dis 2008:14:1390-7.

13. Roxburgh CS, Youngson GG, Townend JA, et al. Trends in pneumonia and empyema in Scottish children in the past 25 years. Arch Dis Child 2008;93:316-18.

14. Li ST, Tancredi DJ. Empyema hospitalizations increased in US children despite pneumococcal conjugate vaccine. Pediatrics 2010;125:26-33.
15. Grijalva CG, Nuorti JP, Zhu Y, et al. Increasing incidence of empyema complicating childhood community-acquired pneumonia in the United States. Clin Infect Dis 2010;50:805-13.

16. Lee GE, Lorch SA, Sheffler-Collins S, et al. National hospitalization trends for pediatric pneumonia and associated complications. Pediatrics 2010;126:204-13

17. Agency for Healthcare Research and Quality. The Healthcare Cost and Utilization Project. Overview of the Nationwide Inpatient Sample. http://www.hcupus.ahrq.gov/nisoverview.jsp (accessed 10 Aug 2010).

18. Agency for Healthcare Research and Quality. The Healthcare Cost and Utilization Project. Hospital Inpatient Statistics, 1996. http://www.hcup-us.ahrq.gov/ reports/natstats/his96/clinclas.htm (accessed 10 Aug 2010).

19. Grijalva CG, Nuorti JP, Arbogast PG, et al. Decline in pneumonia admissions after routine childhood immunisation with pneumococcal conjugate vaccine in the USA: a time-series analysis. Lancet 2007;369:1179-86.

20. Buckingham SC, King MD, Miller ML. Incidence and etiologies of complicated parapneumonic effusions in children, 1996 to 2001. Pediatr Infect Dis $J$ 2003:22:499-504.

21. Pilishvili T, Lexau C, Farley MM, et al. Sustained reductions in invasive pneumococcal disease in the era of conjugate vaccine. J Infect Dis 2010;201:32-41.

22. Poehling KA, Talbot TR, Griffin MR, et al. Invasive pneumococcal disease among infants before and after introduction of pneumococcal conjugate vaccine. JAMA 2006;295:1668-74

23. Tsai CJ, Griffin MR, Nuorti JP, et al. Changing epidemiology of pneumococcal meningitis after the introduction of pneumococcal conjugate vaccine in the United States. Clin Infect Dis 2008;46:1664-72.

24. Whitney CG, Farley MM, Hadler J, et al. Decline in invasive pneumococcal disease after the introduction of protein-polysaccharide conjugate vaccine. N Engl J Med 2003;348:1737-46.

25. Tan TQ, Mason EO Jr, Wald ER, et al. Clinical characteristics of children with complicated pneumonia caused by Streptococcus pneumoniae. Pediatrics 2002;110:1-6.

26. Ampofo K, Herbener A, Blaschke AJ, et al. Association of 2009 pandemic influenza A (H1N1) infection and increased hospitalization with parapneumonic empyema in children in Utah. Pediatr Infect Dis J 2010:29:905-9.

27. Byington CL, Hulten KG, Ampofo K, et al. Molecular epidemiology of pediatric pneumococcal empyema from 2001 to 2007 in Utah. J Clin Microbiol 2010:48:520-5.

28. Maskell NA, Batt S, Hedley EL, et al. The bacteriology of pleural infection by genetic and standard methods and its mortality significance. Am J Respir Crit Care Med 2006;174:817-23.

29. Spellberg B, Guidos R, Gilbert D, et al. The epidemic of antibiotic-resistant infections: a call to action for the medical community from the Infectious Diseases Society of America. Clin Infect Dis 2008;46:155-64.

30. Kyaw MH, Lynfield R, Schaffner W, et al. Effect of introduction of the pneumococca conjugate vaccine on drug-resistant Streptococcus pneumoniae. N Engl J Med 2006;354:1455-63.

31. Anthonisen NR. Empyema. Can Respir J 2008;15:69-70.

32. Avansino JR, Goldman B, Sawin RS, et al. Primary operative versus nonoperative therapy for pediatric empyema: a meta-analysis. Pediatrics 2005;115:1652-9.

33. Eltringham G, Kearns A, Freeman R, et al. Culture-negative childhood empyema is usually due to penicillin-sensitive Streptococcus pneumoniae capsular serotype 1. J Clin Microbiol 2003;41:521-2.

34. Tarrago D, Fenoll A, Sanchez-Tatay D, et al. Identification of pneumococcal serotypes from culture-negative clinical specimens by novel real-time PCR. Clin Microbiol Infect 2008:14:828-34.

35. Ploton C, Freydiere AM, Benito $Y$, et al. Streptococcus pneumoniae thoracic empyema in children: rapid diagnosis by using the Binax NOW immunochromatographic membrane test in pleural fluids. Pathol Biol (Paris) 2006:54:498-501.

36. Le Monnier A, Carbonnelle E, Zahar JR, et al. Microbiological diagnosis of empyema in children: comparative evaluations by culture, polymerase chain reaction, and pneumococcal antigen detection in pleural fluids. Clin Infect Dis 2006;42:1135-40. 